EPJ Web of Conferences 59, 17011 (2013)

DOI: $10.1051 /$ epjconf/20135917011

(C) Owned by the authors, published by EDP Sciences, 2013

\title{
Efficient ion beam generation in laser interactions with micro-structured targets
}

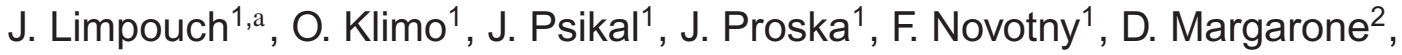 \\ A. Velyhan ${ }^{2}$, M. Cutroneo ${ }^{3}$ and L. Torrisi ${ }^{3}$ \\ 1 Czech Technical University in Prague, FNSPE, Brehova 7, 11519 Praha 1, Czechia \\ 2 Institute of Physics, AS CR, ELI-Beamlines Project, Na Slovance 2, 18221 Praha 8, \\ Czechia \\ 3 INFN, I-95129 Catania, Italy and Univ. Messina, Dept. Physics, I-98166 Messina, Italy
}

\begin{abstract}
The maximum ion energy and acceleration efficiency have to be increased for practical applications of intense ion beams produced by intense short laser pulses incident on a thin foil. For this aim, we propose to use foil with a microscopic structure on the front size. We have prepared such targets by depositing a monolayer of polystyrene nanospheres of a size comparable to laser wavelength on a thin foil. The damage threshold of the produced targets is found experimentally above $3.5 \times 10^{9} \mathrm{~W} / \mathrm{cm}^{2}$ for a nanosecond pedestal and above $10^{11} \mathrm{~W} / \mathrm{cm}^{2}$ for femtosecond prepulses.
\end{abstract}

\section{INTRODUCTION}

Standard mechanism for ion acceleration by short relativistic laser pulse is the target normal sheath acceleration (TNSA) [1]. TNSA is based on laser energy transformation to energetic electrons that penetrate to vacuum at the rear side of a thin foil and produce there a strong quasi-static electric field that accelerates ions from the target surface. For any target material, protons from the deposits on the target surface are preferentially accelerated due to their maximum ion charge to mass ratio. The generated collimated laminar proton beam is used for applications, such as proton imaging and isochoric heating [2]. For most applications, it is desirable to increase the maximum ion energy, as well as the efficiency of laser energy transformation to the accelerated ion beam.

Maximum proton energy and energy transformation have been enhanced in the recent experiments by reducing the foil thickness [3] and lateral dimensions of the target [4]. However, high laser contrast is inevitable for such experiments in order to avoid premature target disruption. Plasma expansion is diminished for high laser contrast, and laser absorption is reduced at very steep plasma profiles. Laser absorption may be boosted by the presence of a nanostructure on the target surface, as was demonstrated experimentally [5] for thick targets. A boost in proton acceleration was observed in thin targets with nanostructure on the front (laser) side via numerical simulations [6] and experimentally [7] for lower intensities. We have proposed to increase the absorption efficiency by using a monolayer of nanospheres on the targets front side [8]. Here, we demonstrate the impact of the nanosphere diameter on laser absorption, proton energy and acceleration efficiency in Section 2. The target fabrication is presented in section 3 together with proton spectra calculated for conditions of experiments under preparation. The conducted target damage tests are described in Section 4.

\footnotetext{
ae-mail: jiri.limpouch@fjfi.cvut.cz
}

This is an Open Access article distributed under the terms of the Creative Commons Attribution License 2.0, which permits unrestricted use, distribution, and reproduction in any medium, provided the original work is properly cited. 

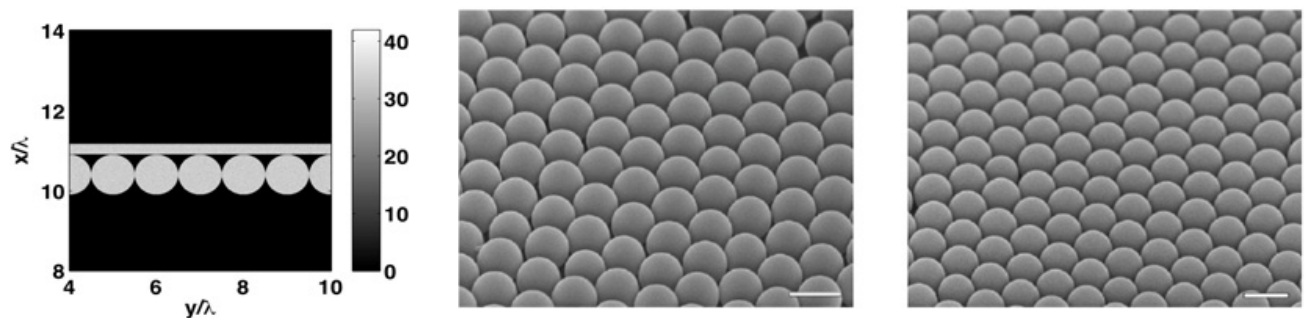

Figure 1. (left) Electron density (normalized on critical density on the color bar) profile in a target used in numerical simulations of proton acceleration. Scanning electron microscope (SEM) images of the target surface of a thin $(900 \mathrm{~nm})$ mylar foil covered by a monolayer of polystyrene spheres with diameter of 920 (middle) and $535 \mathrm{~nm}$ (right) (lower right corners - scale bar $=1 \mu \mathrm{m}$ ).
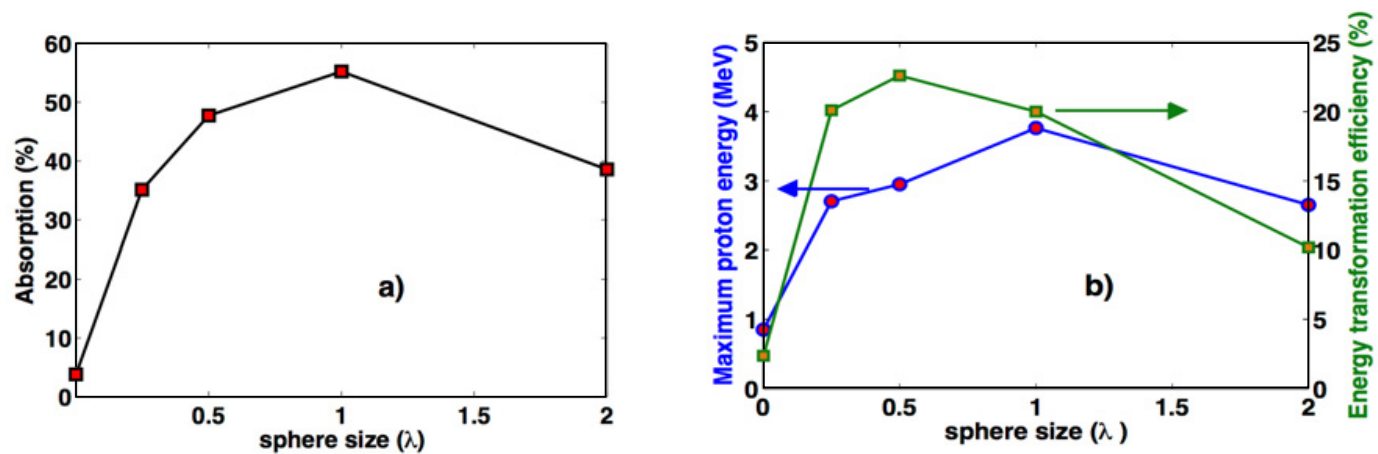

Figure 2. (a) Laser absorption efficiency, (b) maximum proton energy and energy transformation into accelerated protons of energy $>1 \mathrm{MeV}$ versus nanosphere diameter (sphere size $=0$ is flat foil).

\section{NUMERICAL SIMULATIONS OF ION ACCELERATION}

Numerical simulations of the short intense laser pulse interactions with foil targets and of the subsequent ion acceleration are performed using our two-dimensional (2D) electromagnetic relativistic PIC code. The target consists of $0.2 \mu \mathrm{m}$ thick foil, with or without a periodic structure attached to the front, laserirradiated, surface (see Fig. 1). The targets are irradiated by p-polarized laser pulses with a wavelength of $800 \mathrm{~nm}$ and the temporal profile $\sin ^{2}(\pi t / \tau)$, where $\tau$ is the laser pulse duration. A $20 \mathrm{fs}$ long (full width at half maximum - FWHM) laser pulse with a maximum intensity of $1.8 \times 10^{19} \mathrm{~W} / \mathrm{cm}^{2}$ is incident normally on the target.

The laser absorption efficiency (Fig. 2a) maximizes for the sphere diameter near to one laser wavelength $\lambda$ at level more than one order higher than $3.8 \%$ for the flat foil. The energy of protons accelerated normally from the foil rear side maximizes for the same sphere size while the energy transformation to protons with energy $>1 \mathrm{MeV}$ is maximal for a smaller nanosphere size (Fig. 2b).

\section{TARGET FABRICATION AND EXPERIMENTS UNDER PREPARATION}

The method of deposition of the monolayer on the targets' surface is described in a simplified way. A water dispersion of polystyrene microspheres is mixed with ethanol (usually 1:1) and carefully applied on the surface of water in a Petri dish using a glass pipette with a bent tip. Self-assembly at the interface of water/air results in the creation of a compact monolayer of close-packed microspheres. The target is submerged under the monolayer and then lifted up slowly so that the monolayer remains on its upper 


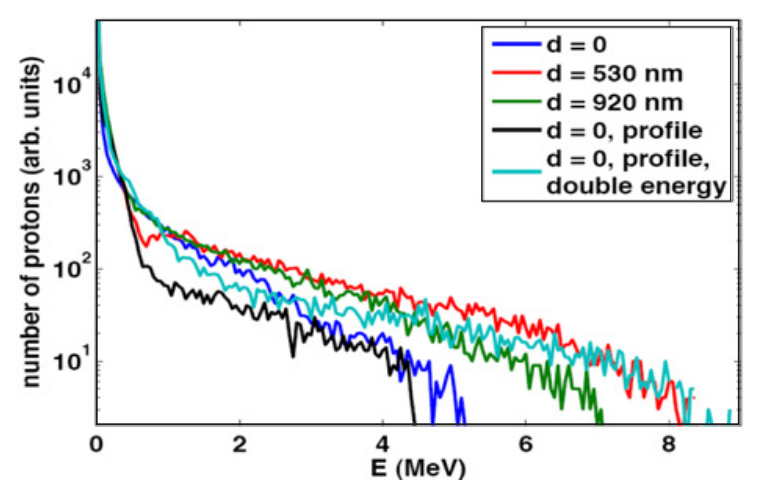

Figure 3. Proton spectra for target without spheres $(\mathrm{d}=0)$ and with spheres of diameter 535 and $920 \mathrm{~nm}$, "profile" in legend corresponds to density scale length $0.1 \lambda$ on laser side of the foil.

side. The result is a piecewise homogeneous monolayer of closely packed microspheres attached to the target surface, which can be seen in Figure 1 (middle and right).

We have performed PIC simulations for planned experiment at the 100 TW laser installation [9] at GIST, Korea, where Ti:Sapphire laser pulse of duration $30 \mathrm{fs}$ incident at the angle $22.5^{\circ}$ is focused to intensity $5 \times 10^{19} \mathrm{~W} / \mathrm{cm}^{2}$ in $3.75 \lambda$ wide Gaussian spot on $900 \mathrm{~nm}$-thick $\mathrm{CH}$ foil either without spheres $(\mathrm{d}=0)$ or with spheres of diameter 535 or $920 \mathrm{~nm}$ on it. For the foil, we have also performed simulations for lower laser contrast when plasma density scale length of $0.1 \lambda$ is assumed on the surface, corresponding to the absence of the double plasma mirror and thus, twice higher energy is available on the target. The proton energy spectra plotted in Fig. 3 reveal that the maximum proton energy for the target with nanospheres of $535 \mathrm{~nm}$ diameter is similar to pure foil irradiated by twice higher laser energy, but the number of accelerated protons is still higher ( $>$ twice higher efficiency).

\section{DAMAGE TESTS OF THE NANOSPHERE TARGETS}

The prepared targets can fulfil their proposed function only if the nanostructure on the surface is not destroyed before the arrival of the main femtosecond laser pulse. However, femtosecond laser pulses are usually preceded by long nanosecond pedestal due to amplified spontaneous emission (ASE), femtosecond prepulses and by picosecond pedestal due to imperfections in pulse compression. The ASE pedestal and prepulses can be damped by special methods, e.g. by using plasma mirrors, but on the expense of the laser complexity and of the laser energy. Thus, the information about the damage threshold of the targets is important for specification of the requirements on the laser contrast.

The damage threshold for the ASE pedestal was estimated by using $9 \mathrm{~ns}$ pulses of Nd-laser [10] at INFN-LNS in Catania. Laser pulse of the energy in range $20-330 \mathrm{~mJ}$, incident at the angle of $30^{\circ}$ on the target, was focused to the spot of diameter $0.5 \mathrm{~mm}$. The laser fluence was in range $10-170 \mathrm{~J} / \mathrm{cm}^{2}$ with the maximum laser intensity of $1.1 \times 10^{9}-1.9 \times 10^{10} \mathrm{~W} / \mathrm{cm}^{2}$. In these experiments, we used targets of a monolayer of polystyrene nanospheres placed on a thick glass slab. The nanosphere diameters were 266, 535 and $920 \mathrm{~nm}$. The irradiated targets were analyzed using optical microscope, scanning electron microscope (SEM) JEOL $7500 \mathrm{~F}$ and atomic force microscope (AFM) Park.

We have not been able to find any spot on the target surface for any nanosphere diameter for laser energy of $165 \mathrm{~mJ}$ or below at fresh target surface, i.e. laser intensity $9.5 \times 10^{9} \mathrm{~W} / \mathrm{cm}^{2}$. Considerable damage on the target surface was observed for all nanosphere diameters for the laser energy $230 \mathrm{~mJ}$, i.e. laser intensity $1.3 \times 10^{10} \mathrm{~W} / \mathrm{cm}^{2}$. Comparison of Fig. 4a) and $4 \mathrm{~b}$ ) demonstrates fast growth of the damaged area with the laser energy. The detail of the damaged edge is presented via SEM (Fig. 4c) and AFM (Fig. 4d) scans. Small increase in the damaged threshold is indicated for the 


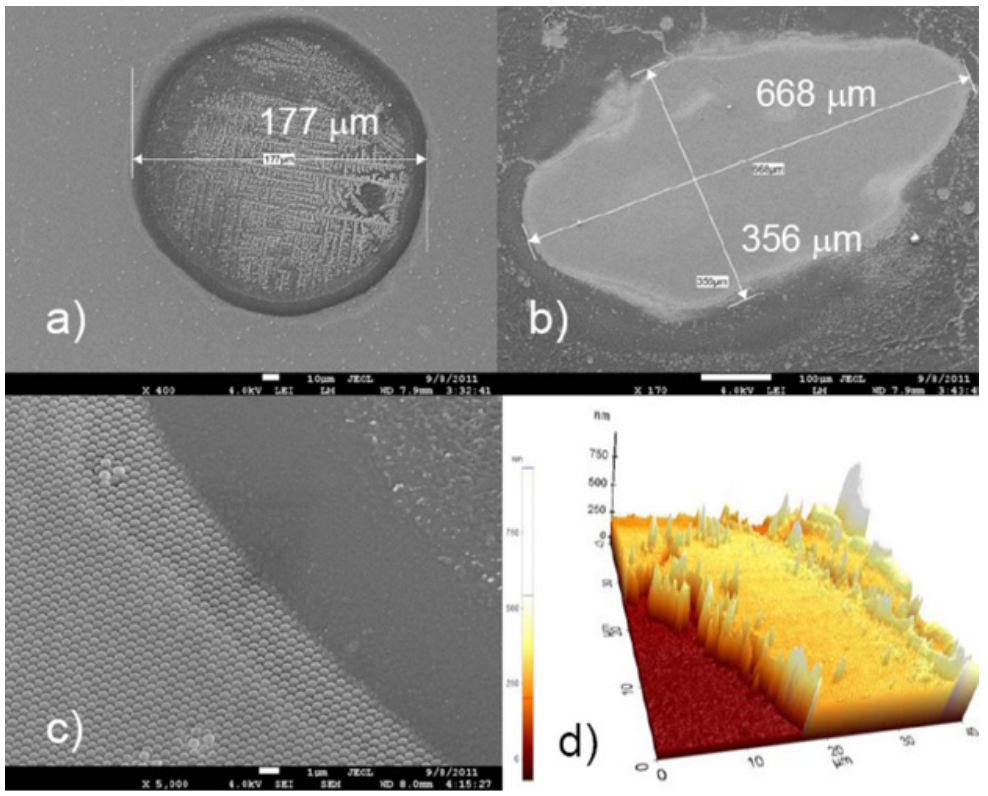

Figure 4. Surface damage for $9 \mathrm{~ns}$ Nd:YAG-pulse incident on target with the monolayer of polystyrene nanospheres of the diameter $535 \mathrm{~nm}$, shot of energy $230 \mathrm{~mJ}$ (a, c, d) and shot of energy $329 \mathrm{~mJ}$ (b). The panels a), b) are overview SEM images, SEM c) a and AFM d) images are details of the spot edge.

layer of small $266 \mathrm{~nm}$ nanospheres, as no damage was observed for the laser energy of $197 \mathrm{~mJ}$ in contrast to the diameter $920 \mathrm{~nm}$. When 4 consecutive shots of energy $165 \mathrm{~mJ}$ are incident at the same spot, the damage is apparent. Thus, it is indicated that the damage threshold $10^{10} \mathrm{~W} / \mathrm{cm}^{2} \mathrm{might}$ be slightly overestimated. Additionally, measurements during the irradiation experiment indicate that weak ion signal was observed above $60 \mathrm{~mJ}$ corresponding to $3.5 \times 10^{9} \mathrm{~W} / \mathrm{cm}^{2}$, but not below. Though nanosecond laser pulse temporal profile differs from ASE pedestal, they can still provide reasonable estimates of the tolerable levels of the ASE pedestal for the targets with nanosphere monolayer.

The tolerated intensity is higher for femtosecond prepulses due to very low fluence. Our preliminary test in Prague has not found any surface damage for $45 \mathrm{fs}$ pulses of intensity $10^{11} \mathrm{~W} / \mathrm{cm}^{2}$.

Double plasma mirror will be applied in experiment to stay below the above damage thresholds.

\section{CONCLUSIONS}

High-quality targets consisting of a thin foil with a monolayer of nanospheres on the laser-irradiated side have been produced. Performed tests show that the requirements on the laser contrast are similar to the thin foil experiments. PIC simulation reveals that such targets enhance the maximum proton energy and the energy conversion into accelerated protons.

Partial support by the Czech Science Foundation (Project P205/11/1165), by the Ministry of Education, Youth and Sports of the Czech Republic through LA0802422, the ELI-Beamlines (CZ.1.05/1.1.00/483/02.0061) and the OPVK (CZ.1.07/2.3.00/20.0087) projects, and by the Grant Agency of the AS CR (project KAN401220801) is gratefully acknowledged. 
IFSA 2011

\section{References}

[1] S.C. Wilks, A.B. Langdon, T.E. Cowan et al. Phys. Plasmas 8, 542 (2001)

[2] M. Borghesi, A. Bigongiari, S. Kar et al., Plasma Phys. Control. Fusion 50, 124040 (2008)

[3] T. Ceccotti, A. Levy, H. Popescu et al., Phys. Rev. Lett. 99, 185002 (2007)

[4] S. Buffechoux, J. Psikal, M. Nakatsumi et al., Phys. Rev. Lett. 105, 015005 (2010)

[5] T. Nishikawa, H. Nakano, K. Oguri et al., J. Appl. Phys. 96, 7537 (2004)

[6] Y. Nodera, S. Kawata, N. Onuma et al., Phys. Rev. E 78, 046401 (2008)

[7] A. Zigler, T. Palchan, N. Bruner et al., Phys. Rev. Lett. 106, 134801 (2011)

[8] O. Klimo, J. Psikal, J. Limpouch et al., New J. Phys. 13, 053028 (2011)

[9] I.J. Kim, T.W. Choi, S.K. Lee et al., Appl. Phys. B-Laser \& Optics 104, 81 (2011)

[10] S. Gammino, L. Torrisi, S. Cavallaro et al., Rev. Sci. Instrum. 81, 02 A508 (2010) 